\title{
A!
}

This is an electronic reprint of the original article.

This reprint may differ from the original in pagination and typographic detail.

Pekola, Jukka P.; Muratore-Ginanneschi, Paolo; Kupiainen, Antti; Galperin, Yuri M.

\section{Energy fluctuations of a finite free-electron Fermi gas}

Published in:

Physical Review E

DOI:

10.1103/PhysRevE.94.022123

Published: 18/08/2016

Document Version

Publisher's PDF, also known as Version of record

Please cite the original version:

Pekola, J. P., Muratore-Ginanneschi, P., Kupiainen, A., \& Galperin, Y. M. (2016). Energy fluctuations of a finite free-electron Fermi gas. Physical Review E, 94(2), 1-4. [022123]. https://doi.org/10.1103/PhysRevE.94.022123

This material is protected by copyright and other intellectual property rights, and duplication or sale of all or part of any of the repository collections is not permitted, except that material may be duplicated by you for your research use or educational purposes in electronic or print form. You must obtain permission for any other use. Electronic or print copies may not be offered, whether for sale or otherwise to anyone who is not an authorised user. 


\title{
Energy fluctuations of a finite free-electron Fermi gas
}

\author{
Jukka P. Pekola, ${ }^{1}$ Paolo Muratore-Ginanneschi, ${ }^{2}$ Antti Kupiainen, ${ }^{2}$ and Yuri M. Galperin ${ }^{3,4}$ \\ ${ }^{1}$ Low Temperature Laboratory, Department of Applied Physics, Aalto University School of Science, P.O. Box 13500, 00076 Aalto, Finland \\ ${ }^{2}$ University of Helsinki, Department of Mathematics and Statistics, P.O. Box 68, FIN-00014 Helsinki, Finland \\ ${ }^{3}$ Department of Physics, University of Oslo, PO Box 1048, Blindern, 0316 Oslo, Norway \\ ${ }^{4}$ Ioffe Institute, 26 Politekhnicheskaya, St. Petersburg 194021, Russian Federation
}

(Received 24 May 2016; published 18 August 2016)

\begin{abstract}
We discuss the energy distribution of free-electron Fermi-gas, a problem with a textbook solution of Gaussian energy fluctuations in the limit of a large system. We find that for a small system, characterized solely by its heat capacity $C$, the distribution can be solved analytically, and it is both skewed and it vanishes at low energies, exhibiting a sharp drop to zero at the energy corresponding to the filled Fermi sea. The results are relevant from the experimental point of view, since the predicted non-Gaussian effects become pronounced when $C / k_{\mathrm{B}} \lesssim 10^{3}$ ( $k_{\mathrm{B}}$ is the Boltzmann constant), a regime that can be easily achieved for instance in mesoscopic metallic conductors at sub-kelvin temperatures.
\end{abstract}

DOI: 10.1103/PhysRevE.94.022123

\section{INTRODUCTION}

Physical quantities of an equilibrium macroscopic system are well characterized by their average values. However, random deviations from the average values-fluctuationsare very important since they contain important information on the system. Under most circumstances the distribution of small fluctuations is Gaussian [1], Chap. 7. However, this is not the case for nanometer-sized devices, which are currently studied intensively [2,3], see [4-7] for a review. Energy and temperature fluctuations in a single electron box [8] were considered in [9]; several works experimental and theoretical, e.g., [10-12], were devoted to temperature fluctuations. Such fluctuations are particularly relevant for ultra-small calorimeters aiming at extreme energy resolution, ultimately down to single microwave photon level [10,13-15]. The effective temperature of the calorimeter, which has heat capacity $C$, changes by $E / C$ upon absorbing a photon of energy $E$. The resolution, $\propto \sqrt{C}$, where $C$ is the heat capacity of the absorber of the calorimeter, needs thus to be minimized. Yet the picture gets more complicated when the absorber is very small (low $C$ ), and the energy and effective temperature of the absorber cannot be considered constant, but they exhibit significant intrinsic fluctuations $[1,9,12]$. It is the purpose of this work to address the problem of energy fluctuations for a particular system of a metallic calorimeter representing a textbook model of free electron gas at low temperatures $T$ far below the Fermi temperature $T_{\mathrm{F}}$. In a typical experimental setting, $T / T_{\mathrm{F}} \sim 10^{-6}[14-16]$.

In the present work we will derive the distribution function for a finite Fermi gas and show that its shape is determined by only one dimensionless parameter-the heat capacity divided by the Boltzmann constant, $k_{\mathrm{B}}$. At $C / k_{\mathrm{B}} \gg 1$ we recover the well-known Gaussian distribution, while at finite values of this parameter significant deviations are expected. We will analytically derive the distribution of the energies of a finite sample of the Fermi gas kept at a given temperature and analyze its properties including moments and skewness absent in the thermodynamic limit. Since the heat capacity of a metallic conductor of sub-micron dimensions at standard sub-kelvin $(\sim 10-100 \mathrm{mK})$ experimental temperatures is of the order of $\left(10^{2}-10^{3}\right) k_{\mathrm{B}}[4,13]$, our results have potential impact on sensitive bolometers [4,10,14-17]. In future, we plan to use the obtained distribution for analysis of heat exchange between a quantum device, e.g., a qubit, and a mesoscopic metallic calorimeter.

The paper is organized as follows. We derive general expression for the energy distribution in Sec. II A, which will be analyzed in Sec. II B. Moments of the distribution are considered in Sec. II C.

\section{ENERGY DISTRIBUTIONS}

\section{A. General expressions}

It is convenient to calculate the Fourier transform, $F(\lambda)$, of the energy distribution function. It can be expressed in the form

$$
F(\lambda)=\left\langle e^{i \lambda E}\right\rangle=\frac{\sum_{m} e^{(i \lambda-\beta) E_{m}}}{\sum_{m} e^{-\beta E_{m}}}=\frac{Z(\beta-i \lambda)}{Z(\beta)}
$$

Here,

$$
Z(\beta) \equiv \sum_{m} e^{-\beta E_{m}}
$$

is the partition function, $\beta \equiv\left(k_{\mathrm{B}} T\right)^{-1}$ where $T$ is temperature, while

$$
E_{m}=\sum_{\mathbf{k}}\left(\epsilon_{\mathbf{k}, m}-\mu\right) n_{\mathbf{k}, m} .
$$

A micro-state is characterized by the set of quantum numbers $\{\mathbf{k}, m\}$ where $\mathbf{k}$ is the quasi-momentum. In the following, concentrating on mesoscopic electronic systems, we will consider a gas of elementary particles characterized only by the quantum number $\mathbf{k}$. Since we are interested in relatively large systems with $10^{7}-10^{8}$ electrons, we will ignore the energy quantization and consider $\mathbf{k}$ as a continuous variable, see estimates in the end of Sec. II B.

According to the definitions (1)-(3), we calculate the characteristic function of quasiparticle energy. The energy distribution function, $P(E)$, can be calculated as the inverse 
Fourier transform of $F(\lambda)$ as

$$
P(E)=\int_{-\infty}^{\infty} \frac{d \lambda}{2 \pi} e^{-i \lambda E} F(\lambda) .
$$

For the Fermi gas [1],

$$
\begin{aligned}
\ln Z(\beta) & =\sum_{\mathbf{k}} \ln \left(1+e^{-\beta\left(\epsilon_{\mathbf{k}}-\mu\right)}\right) \\
& =\mathcal{V} \int_{0}^{\infty} d \epsilon N(\epsilon) \ln \left(1+e^{-\beta(\epsilon-\mu)}\right) .
\end{aligned}
$$

Here, $N(\epsilon)$ is the density of states while $\mathcal{V}$ is the volume of the system. In the following we put $N(\epsilon)=\mathrm{const} \equiv N_{0}$. This assumption is valid for the two-dimensional gas, while in the three-dimensional case $N(\epsilon) \propto \sqrt{\epsilon}$. One can show that in the limiting case $\beta \mu \gg 1$ the results differ only by renormalization of numerical constants in the expressions for the average energy and heat capacity. For realistic experimental conditions involving typical metals $\beta \mu=T_{\mathrm{F}} / T \sim 10^{6}$-for a metal with Fermi temperature $T_{\mathrm{F}}=10^{5} \mathrm{~K}$ at $T=0.1 \mathrm{~K}$. The situations where $N(\epsilon)$ is a rapidly varying function, such as resonant scattering or Kondo effect require special treatment, we are not considering in this paper.

For $N(\epsilon)=N_{0}$ we obtain

$$
\begin{aligned}
\ln Z(\beta) & =\frac{\mathcal{V} N_{0}}{\beta} \int_{-\beta \mu}^{\infty} d \xi \ln \left(1+e^{-\xi}\right) \\
& =-\frac{\mathcal{V} N_{0}}{\beta} \operatorname{dilog}\left(1+e^{\beta \mu}\right) .
\end{aligned}
$$

Here,

$$
\operatorname{dilog}(x)=\int_{1}^{x} \frac{\ln (t)}{1-t} d t
$$

is the dilogarithm function. At $\beta \mu \gg 1$ we expand

$$
-\operatorname{dilog}\left(1+e^{\beta \mu}\right)=\frac{1}{2}(\beta \mu)^{2}+\frac{\pi^{2}}{6}-e^{-\beta \mu}+\ldots
$$

Keeping two first terms in the r.h.s. of Eq. (9) we obtain

$$
\ln Z(\beta)=\mathcal{V} N_{0}\left(\frac{1}{2} \beta \mu^{2}+\frac{\pi^{2}}{6} \frac{1}{\beta}\right) .
$$

Note that we assume that the chemical potential is fixed rather than determined by the normalization of the singleparticle distribution to the total number of particles. This assumption is appropriate for an electronic system with contacts.

In the case when $N(\epsilon) \propto \sqrt{\epsilon}$ we can put $N(\epsilon)=N_{0} \sqrt{\epsilon / \mu}$ to obtain instead of Eq. (10)

$$
\ln Z(\beta)=\mathcal{V} N_{0}\left(\frac{2}{5} \beta \mu^{2}+\frac{\pi^{2}}{4} \frac{1}{\beta}\right) .
$$

Therefore, different forms of the dependence of the density of states upon the energy indeed result only in numerical constants defining the average energy and heat capacity. The difference in the numerical factors is compatible with the well-known Sommerfeld expansion, see, e.g., [18].
Using the expansion (10) we obtain

$$
\begin{aligned}
\ln F(\lambda) & =\ln \frac{Z(\beta-i \lambda)}{Z(\beta)} \\
& =\frac{\mathcal{V} N_{0}}{2}\left(-i \lambda \mu^{2}+\frac{\pi^{2}}{3} \frac{i \lambda}{\beta(\beta-i \lambda)}\right) .
\end{aligned}
$$

The first item is responsible for the energy shift of the energy by the Fermi energy $E_{0}=\mathcal{V} N_{0} \mu^{2} / 2$. In our approximation $(\beta \mu \gg 1)$ the specific heat has the standard free Fermi-gas expression (for a given chemical potential)

$$
C=k_{\mathrm{B}} \frac{\pi^{2}}{3 \beta} N_{0} \mathcal{V} \text {. }
$$

Then the quantity in the parentheses in Eq. (12) can be rewritten as

$$
i \lambda E_{0}+\frac{C}{2 k_{\mathrm{B}}} \frac{i \lambda}{\beta-i \lambda} .
$$

We rewrite

$$
\begin{aligned}
\frac{C}{2 k_{\mathrm{B}}} \frac{i \lambda}{\beta-i \lambda} & =i \lambda \frac{C}{2 k_{\mathrm{B}} \beta}\left(\frac{i \lambda}{\beta-i \lambda}+1\right)-i \lambda \frac{C}{2 k_{\mathrm{B}} \beta} \\
& \equiv-i \lambda E_{\beta}+\frac{C}{2 k_{\mathrm{B}} \beta} \frac{(i \lambda)^{2}}{\beta-i \lambda} .
\end{aligned}
$$

Here, $E_{\beta}=C / 2 k_{\mathrm{B}} \beta$. Denoting $\delta E=E-E_{0}-E_{\beta}$ we obtain in the exponent

$$
-i \lambda \delta E-\frac{C}{2 k_{\mathrm{B}}} \frac{\lambda^{2}}{\beta(\beta-i \lambda)} .
$$

Let us measure the energy deviation $\delta E$ in units of $\beta^{-1} \sqrt{C / k_{\mathrm{B}}}$. Then we put

$$
\lambda \equiv \frac{\beta}{\sqrt{C / k_{\mathrm{B}}}} \Lambda, \quad \delta E \equiv \frac{\sqrt{C / k_{\mathrm{B}}}}{\beta} u, \quad c \equiv \frac{C}{k_{\mathrm{B}}}
$$

to obtain for the second term

$$
-\frac{1}{2} \frac{\Lambda^{2}}{1-i \Lambda / \sqrt{c}}=-\frac{1}{2} \frac{\Lambda^{2}(1+i \Lambda / \sqrt{c})}{1+\Lambda^{2} / c} .
$$

Therefore, at $\beta \mu \gg 1$ we arrive at the expression for the distribution of dimensionless energies $u$ :

$$
P(u)=\int_{-\infty}^{\infty} \frac{d \Lambda}{2 \pi} \exp \left[i \Lambda\left(\frac{1}{2} \frac{i \Lambda}{1-i \Lambda / \sqrt{c}}-u\right)\right] .
$$

This is our final expression, which we have to analyze for different values of dimensionless heat capacity, $c$.

\section{B. Energy distributions for different dimensionless heat capacity}

We compute the integral in Eq. (19) by deforming the contour in the complex plane. The integrand is analytic in the full complex plane except for (an essential) singularity at $\Lambda=-i \sqrt{c}$. For large $|\Lambda|$ the argument of the exponential behaves as

$$
-\frac{1}{2} \frac{\Lambda^{2}}{1-i \Lambda / \sqrt{c}}-i \Lambda u=-\frac{c}{2}-i \Lambda\left(\frac{\sqrt{c}}{2}+u\right)+\mathcal{O}(1) .
$$

For thermodynamics it suffices to consider energies above the Fermi energy $E>E_{0}$ which means $u+\sqrt{c} / 2>0$. We may 
then deform the contour to the lower half-plane $\operatorname{Im} \Lambda<0$. We get

$$
P(u)=P_{0}(u)+P_{\infty}(u),
$$

where $P_{\infty}(u)$ is contribution from the semi circle at infinity in the lower half-plane and $P_{0}(u)$ from a circle around $\Lambda=-i \sqrt{c}$.

Writing $\Lambda=\rho e^{i \phi}, \pi \leqslant \phi \leqslant 2 \pi$ we obtain $P_{\infty}(u)$ as the limit of

$$
e^{-\frac{1}{2} c} \frac{i \rho}{2 \pi} \int_{2 \pi}^{\pi} d \phi e^{i\left(\phi-\rho b e^{i \phi}\right)}=\frac{\sin (b \rho)}{\pi b}
$$

as $\rho \rightarrow \infty$, i.e., $P_{\infty}(u)$ where $b=\sqrt{c} / 2+u$. Since we assume $b \neq 0$ this limit vanishes. get

To compute $P_{0}(u)$ change variables to $z=\frac{1}{c}\left(1-i \frac{\Lambda}{\sqrt{c}}\right)$ to

$$
P_{0}(u)=c^{\frac{3}{2}} e^{-(2 c+\sqrt{c} u)} \oint e^{\frac{1}{z}} e^{x z} \frac{d z}{2 \pi i},
$$

where $x=c(c+\sqrt{c} u)$ and the integral is around the origin. Then

$$
\begin{aligned}
\oint e^{\frac{1}{z}} e^{x z} \frac{d z}{2 \pi i} & =\sum_{n=0}^{\infty} \frac{1}{n !} \oint z^{-n} e^{x z} \frac{d z}{2 \pi i} \\
& =\sum_{m=0}^{\infty} \frac{1}{(m+1) !} \frac{1}{m !} x^{m}=x^{-\frac{1}{2}} I_{1}\left(2 x^{\frac{1}{2}}\right),
\end{aligned}
$$

where

$$
I_{1}(z)=\frac{1}{\pi} \int_{0}^{\pi} e^{z \cos \theta} \cos \theta d \theta
$$

is the modified Bessel function of first kind [19].

As a result, at $\beta \mu \gg 1$

$$
P(u)=\frac{c^{3 / 4}}{\sqrt{\sqrt{c}+2 u}} I_{1}\left(c^{3 / 4} \sqrt{\sqrt{c}+2 u)} e^{-u \sqrt{c}-c} .\right.
$$

Graphs for $P(u)$ for different heat capacities, $c$, are shown in Fig. 1. One observes that at large $c$ the distribution is essentially Gaussian. That can be found analytically-using the asymptotic expression $I_{1}(x) \rightarrow(2 \pi x)^{-1 / 2} e^{x}$ for $x \gg 1$. One then arrives at the expression

$$
P(u)=\frac{1}{\sqrt{2 \pi}} e^{-u^{2} / 2} .
$$

In the opposite limit, at small $c$ it becomes very asymmetric.

Let us crudely estimate the dimensionless heat capacity, $c$. The estimate reads as $c \sim n_{e} \mathcal{V}\left(k_{\mathrm{B}} T / \mu\right)$, where $n_{e}$ is the electron density. For a typical metal $n_{e} \approx 10^{23} \mathrm{~cm}^{-3}$. Assuming the sizes of a mesoscopic system (in $\mu \mathrm{m}$ ) as $(0.3-1) \times(0.05-0.1) \times(0.01-0.03)(\mu \mathrm{m})^{3}$, we get

$$
\mathcal{V}=\left(1.5 \times 10^{-16}-3 \times 10^{-15}\right) \mathrm{cm}^{3}
$$

and the total number of electrons is

$$
\mathcal{N} \equiv n_{e} \mathcal{V}=1.5 \times 10^{7}-3 \times 10^{8} .
$$

These numbers are motivated by the dimensions of top-down nanostructures fabricated, e.g., by electron-beam lithography and metal deposition with lift-off, see basic examples in Refs. [15,20], and even smaller metallic dots achieved by

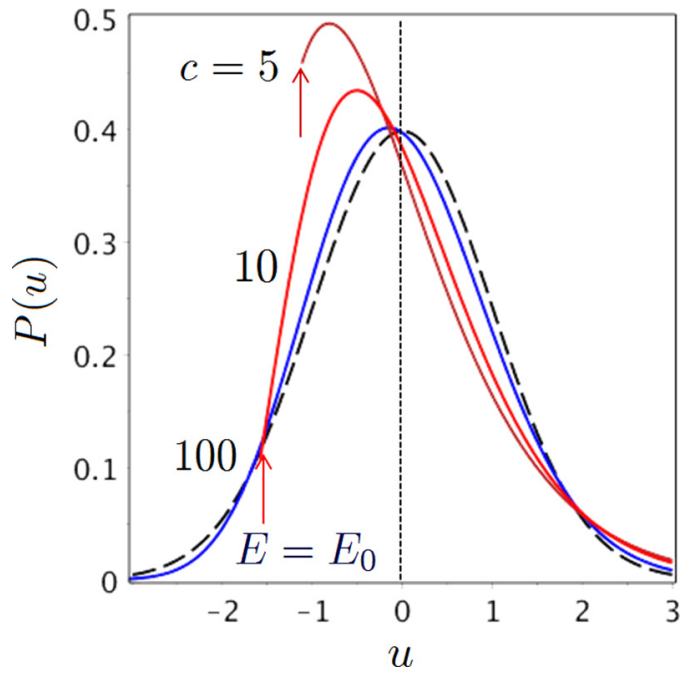

FIG. 1. Distribution $P(u)$, where $u=\beta \delta E / \sqrt{c}$, where $\delta E$ is the deviation of the energy of the Fermi-gas from its mean, for a few different values of $c=C / k_{\mathrm{B}}$ (solid lines). Dashed line is the Gaussian distribution.

this technique in Ref. [21]. Putting $T_{\mathrm{F}} \approx 5 \times 10^{4} \mathrm{~K}$ and $T=$ (0.05-0.1) K we get

$$
k_{\mathrm{B}} T / \mu=T / T_{\mathrm{F}}=(1-2) \times 10^{-6} .
$$

Consequently, the range of the quantity $c$ is $15-600$. As we have seen, at these values of $c$ the energy distribution is clearly non-Gaussian.

Note that in the course of the derivation of the distribution function we assumed continuous electron energy spectrum. Generally, it is not the case in a finite Fermi system. In realistic metallic grains the inter-level spacings can be considered as random, see [22,23] for a review. The mean level spacing can be estimates as $1 / N_{0} \mathcal{V}$ that is $\lesssim 1 \mathrm{mK}$ for the grains we consider. Since we are interested in higher temperatures the model of continuous electron spectrum can be used.

\section{Moments of the energy distribution}

The moments of the energy distribution can be readily calculated either from Eq. (19), or explicitly from the Hamiltonian $\mathcal{H}=\sum_{\mathbf{k}} \epsilon_{\mathbf{k}} a_{\mathbf{k}}^{\dagger} a_{\mathbf{k}}$. In particular,

$$
\langle E\rangle=\sum_{\mathbf{k}} \epsilon_{\mathbf{k}}\left\langle a_{\mathbf{k}}^{\dagger} a_{\mathbf{k}}\right\rangle=\sum_{\mathbf{k}} \epsilon_{\mathbf{k}} f\left(\epsilon_{\mathbf{k}}\right),
$$

where $f(\epsilon)=\left[e^{\beta(\epsilon-\mu)}+1\right]^{-1}$ is the Fermi function. The second moment reads

$$
\begin{aligned}
\left\langle E^{2}\right\rangle & =\sum_{\mathbf{k}} \epsilon_{\mathbf{k}} \epsilon_{\mathbf{l}}\left\langle a_{\mathbf{k}}^{\dagger} a_{\mathbf{k}} a_{\mathbf{l}}^{\dagger} a_{\mathbf{l}}\right\rangle \\
& =\langle E\rangle^{2}+\sum_{\mathbf{k}} \epsilon_{\mathbf{k}}^{2} f\left(\epsilon_{\mathbf{k}}\right)\left[1-f\left(\epsilon_{\mathbf{k}}\right)\right] .
\end{aligned}
$$

Here, we have decomposed the product of four Fermi operators according to the Wick theorem. 
We observe that only the vicinity of the Fermi level is important for the difference

$$
\left\langle\delta E^{2}\right\rangle \equiv\left\langle(E-\langle E\rangle)^{2}\right\rangle=\left\langle E^{2}\right\rangle-\langle E\rangle^{2} .
$$

Therefore, while calculating $\left\langle\delta E^{2}\right\rangle$ we can assume constant density of states and $\beta \mu \gg 1$. In this way we obtain

$$
\left\langle\delta E^{2}\right\rangle=k_{\mathrm{B}} T^{2} C
$$

In a similar way, we calculate

$$
\left\langle\delta E^{3}\right\rangle=2 \sum_{\mathbf{k}} \epsilon_{\mathbf{k}}^{3} f\left(\epsilon_{\mathbf{k}}\right)\left[1-f\left(\epsilon_{\mathbf{k}}\right)\right]^{2}=3 k_{\mathrm{B}}^{2} T^{3} C .
$$

The skewness of the distribution is then

$$
\gamma=\left\langle\delta E^{3}\right\rangle /\left\langle\delta E^{2}\right\rangle^{3 / 2}=3 / \sqrt{c}
$$

Obviously, the skewness vanishes as $c \rightarrow \infty$ which is the thermodynamic limit. For a Gaussian distribution, the relationship between the second and the fourth moments is $\left\langle\delta E^{4}\right\rangle=$ $3\left\langle\delta E^{2}\right\rangle^{2}$. In the general case, an additional contribution appears, such that,

$$
\left\langle\delta E^{4}\right\rangle=3\left\langle\delta E^{2}\right\rangle^{2}\left[1+\frac{4}{\sqrt{c}}\right] .
$$

The fifth moment, absent in the Gaussian approximation, is

$$
\left\langle\delta E^{5}\right\rangle=60 k_{\mathrm{B}}^{4} T^{5} C .
$$

The results obtained by numerical integration of Eq. (19) agree with the analytic expressions given above.

In summary, we have shown that the energy distribution of a free Fermi-gas with small heat capacity is non-Gaussian, with a sharp cut-off at low energies. This is a natural consequence of the minimal energy of the filled Fermi sea. We find that the heat capacities demonstrating strong non-Gaussian features can be achieved in standard metallic nanodevices at sub-kelvin temperatures.

\section{ACKNOWLEDGMENTS}

We thank Ivan Khaymovich and Kay Schwieger for discussions. Y.M.G. thanks Aalto University for hospitality during the preparation of this manuscript. The work has been supported by the Academy of Finland (Contracts No. 272218, No. 284594, and No. 271983).
[1] L. D. Landau and E. M. Lifshitz, Statistical Physics, Part 1 (Pergamon Press, Oxford, 1980).

[2] C. Jarzynski, Phys. Rev. Lett. 78, 2690 (1997).

[3] G. E. Crooks, Phys. Rev. E 60, 2721 (1999).

[4] F. Giazotto, T. T. Heikkilä, A. Luukanen, A. M. Savin, and J. P. Pekola, Rev. Mod. Phys. 78, 217 (2006).

[5] Thermoelectric Handbook, Macro to Nano, edited by D. M. Rowe (Taylor and Francis, London, 2006).

[6] M. Campisi, P. Hänggi, and P. Talkner, Rev. Mod. Phys. 83, 771 (2011).

[7] U. Seifert, Rep. Prog. Phys. 75, 126001 (2012).

[8] G. L. Ingold and Y. V. Nazarov, in Single Charge Tunneling (NATO ASI Series B 294) edited by H. Garbert and M. Devoret (Plenum, New York, 1992).

[9] T. L. van den Berg, F. Brange, and P. Samuelsson, New J. Phys. 17, 075012 (2015).

[10] S. Gasparinetti, K. L. Viisanen, O.-P. Saira, T. Faivre, M. Arzeo, M. Meschke, and J. P. Pekola, Phys. Rev. Appl. 3, 014007 (2015).

[11] K. L. Viisanen, S. Suomela, S. Gasparinetti, O.-P. Saira, J. Ankerhold, and Jukka. P. Pekola, New. J. Phys. 17, 055014 (2015).

[12] T. T. Heikkilä and Y. V. Nazarov, Phys. Rev. Lett. 102, 130605 (2009).
[13] J. P. Pekola, P. Solinas, A. Shnirman, and D. V. Averin, New J. Phys. 15, 115006 (2013).

[14] J. Govenius, R. E. Lake, K. Y. Tan, V. Pietilä, J. K. Julin, I. J. Maasilta, P. Virtanen, and M. Möttönen, Phys. Rev. B 90, 064505 (2014).

[15] O.-P. Saira, M. Zgirski, K. L. Viisanen, D. S. Golubev, and J. P. Pekola, arXiv:1604.05089.

[16] K. L. Viisanen and J. P. Pekola, arXiv:1606.02985.

[17] P. L. Richards, J. Appl. Phys. 76, 1 (1994).

[18] A. L. Fetter and J. D. Walecka, Quantum Theory of ManyParticle Systems (McGraw-Hill Book Company, New York, 1971), p. 47.

[19] Modified Bessel Functions I and K, in Handbook of Mathematical Functions with Formulas, Graphs, and Mathematical Tables, 9th printing, edited by M. Abramowitz and I. A. Stegun (Dover, New York, 1972), pp. 374-377.

[20] J. P. Pekola, O.-P. Saira, V. F. Maisi, A. Kemppinen, M. Möttönen, Yu. A. Pashkin, and D. V. Averin, Rev. Mod. Phys. 85, 1421 (2013).

[21] Yu. A. Pashkin, Y. Nakamura, and J. S. Tsai, Appl. Phys. Lett. 76, 2256 (2000).

[22] W. P. Halperin, Rev. Mod. Phys. 58, 533 (1986).

[23] I. S. Beloborodov, A. V. Lopatin, V. M. Vinokur, and K. B. Efetov, Rev. Mod. Phys. 79, 469 (2007). 